\title{
Usability Testing of Gaming and Social Media Applications for Stroke and Cerebral Palsy Upper Limb Rehabilitation
}

\author{
Bulmaro A. Valdés, Student Member, IEEE, Courtney G. E. Hilderman, Chai-Ting Hung, Navid \\ Shirzad, H.F. Machiel Van der Loos, Member, IEEE
}

\begin{abstract}
As part of the FEATHERS (Functional Engagement in Assisted Therapy Through Exercise Robotics) project, two motion tracking and one social networking applications were developed for upper limb rehabilitation of stroke survivors and teenagers with cerebral palsy. The project aims to improve the engagement of clients during therapy by using video games and a social media platform. The applications allow users to control a cursor on a personal computer through bimanual motions, and to interact with their peers and therapists through the social media. The tracking applications use either a Microsoft Kinect or a PlayStation Eye camera, and the social media application was developed on Facebook. This paper presents a usability testing of these applications that was conducted with therapists from two rehabilitation clinics. The "Cognitive Walkthrough" and "Think Aloud" methods were used. The objectives of the study were to investigate the ease of use and potential issues or improvements of the applications, as well as the factors that facilitate and impede the adoption of technology in current rehabilitation programs.
\end{abstract}

\section{INTRODUCTION}

Hemiplegia, a movement disorder affecting one side of the body, impacts the daily functioning and participation of many children with cerebral palsy (CP) and adults after stroke. These individuals often demonstrate impairments in upper limb sensation, range of motion, strength, control and coordination [1], [2]. Functional limitations include the ability of the individuals to perform bimanual tasks such as carrying a food tray. In rehabilitation, intensive practice of bimanual motions is used to promote the functional recovery of an individual's hemiplegic arm. The engagement of a client in therapy impacts functional outcomes, and motivating interventions, such as virtual reality applications, are expected to improve clients' engagement [3], [4]. The "Functional Engagement in Assisted Therapy Through Exercise Robotics" project [5], investigates the combination of virtual reality and social networking as a motivating at-home therapy, providing an opportunity for intensive practice of bimanual motions. The target populations for this project are hemiplegic stroke survivors and teenagers with cerebral palsy.

\section{METHODS}

Three computer applications were developed to allow users to play video games on Facebook in the private

Research supported by the Peter Wall Solutions Initiative.

B.A. Valdés, C.T. Hung, N. Shirzad, and H.F.M. Van der Loos are with the Department of Mechanical Engineering, and C.G.E. Hilderman is with the Department of Physical Therapy, of the University of British Columbia, Vancouver, BC, V6T 1Z4, Canada (phone: 604-822-3147; fax: 604-822-2403; e-mail: bulmaro.valdes@alumni.ubc.ca).

(C) 2014 IEEE. Personal use of this material is permitted. Permission from IEEE must be obtained for all other uses, in any current or future media, including reprinting/republishing this material for advertising or promotional purposes, creating new collective works, for resale or redistribution to servers or lists, or reuse of any copyrighted component of this work in other works.
FEATHERS social group. One application allows interactions between users, and between users and their therapists. Two applications track the users' bimanual motions and map them to a computer cursor for playing video games.

In this phase of the project, we conducted a usability test on the developed applications using the "Think Aloud" and "Cognitive Walkthrough" methods. The subjects were rehabilitation professionals. This study aimed to assess the ease of use of the applications and their interfaces, identify potential issues or improvements, and investigate the factors that might facilitate and impede the use of this type of technology in rehabilitation. The next phase of the FEATHERS project involves user testing with subjects from the target populations. This will precede a 6-month clinical trial in which subjects will use the systems at home.

\section{A. System Description}

Two of the FEATHERS applications use commercially available motion tracking technology (Microsoft Kinect ${ }^{\mathrm{TM}}$, and PlayStation ${ }^{\circledR}$ Eye camera and Move controllers) to map the users' bimanual motions on personal computers running Microsoft Windows. The Kinect camera tracks users' movements without additional equipment, while the PlayStation Eye camera requires the use of two Move controllers for tracking arm motions. For the usability test, two customized controllers (Fig. 1) were used along with the PlayStation Eye camera.

Our novel interface requires the user to move both arms to interact with a computer, thus avoiding the reliance on the unaffected side to rehabilitate at home. To operate the system the user has to stand two meters away from the tracking camera and use hand movements and gestures to perform the basic functions of the computer's mouse/trackpad. Two bimanual input modes are available: Visual Symmetry (VS) and Point Mirror Symmetry (PMS) [6]. Using the VS mode requires users to move both hands in the same direction at the same time, while PMS requires users to move both hands in a circular manner that is similar to steering a wheel. To promote the use of the weak side, the displacement vectors of the

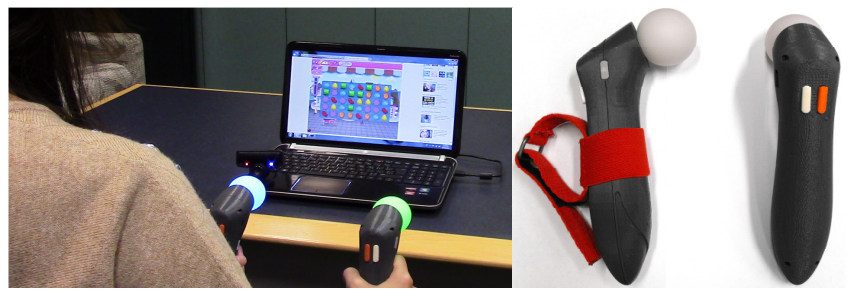

Fig. 1. Customized Controllers 
paretic and non-paretic hands are compared on each iteration of the program, and the one with the smallest magnitude is mapped to the motion of the computer's cursor. Both applications have a Graphical User Interface (GUI) that enables the user to change the following parameters: bimanual tracking mode, range of motion, data logging, and specific options for each tracking technology. The GUI for the Kinect and PlayStation applications are shown in Fig. 2, and Fig. 3, respectively.

The chosen social media platform was Facebook (Fig. 4) since it supports user interactions and provides a large game library that can accommodate individual preferences. The application serves as a channel for communication between users and their therapists, provides a list of therapists' recommended games, and registers scores from previously played games. The user also has the option to join a Facebook group, which includes other users of the application, to promote socialization through the sharing of posts.

\section{B. Subjects}

This study recruited 11 rehabilitation professionals (10 females, 1 male) who were specialized in stroke and cerebral palsy care. There were 9 physiotherapists and 2 occupational therapists, with an average of 11.45 years of experience, ranging from 1 to 20 years. There were five subjects between the ages of 20-34, four between 35-49, and two between 50-64. The sessions were conducted at two rehabilitation

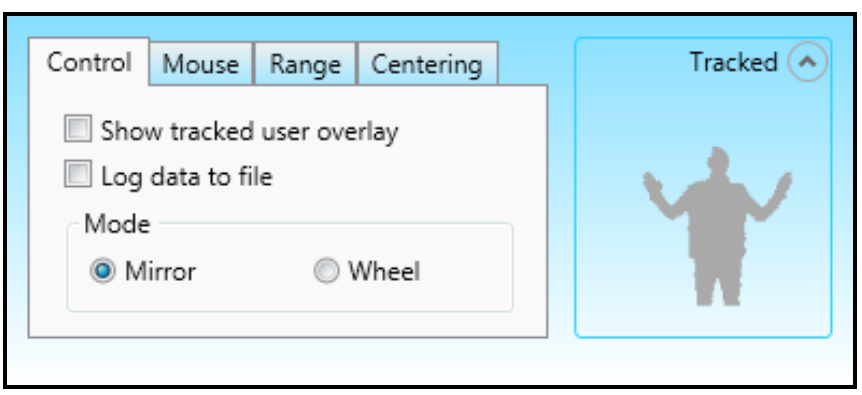

Fig. 2. Kinect Application

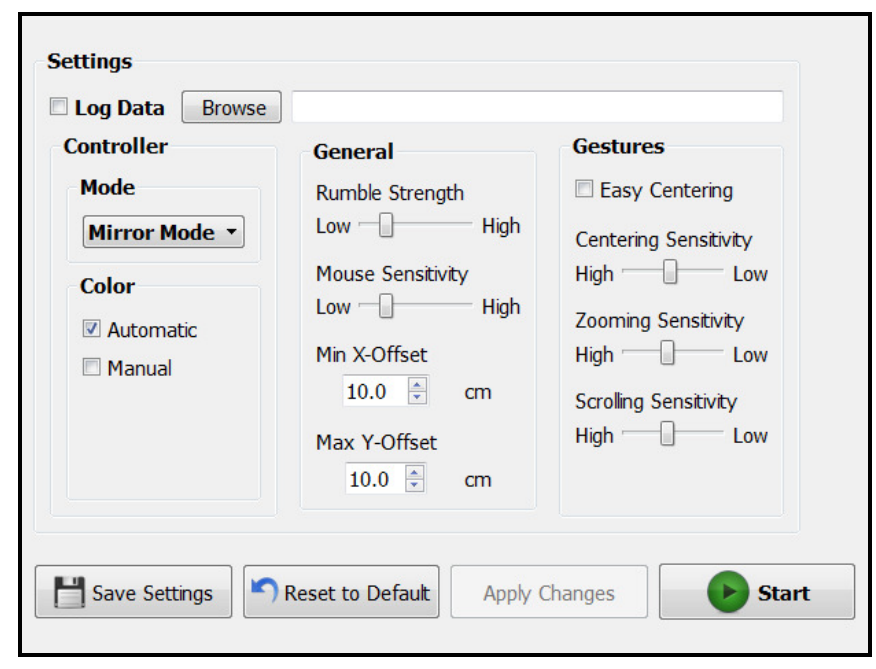

Fig. 3. PlayStation Application

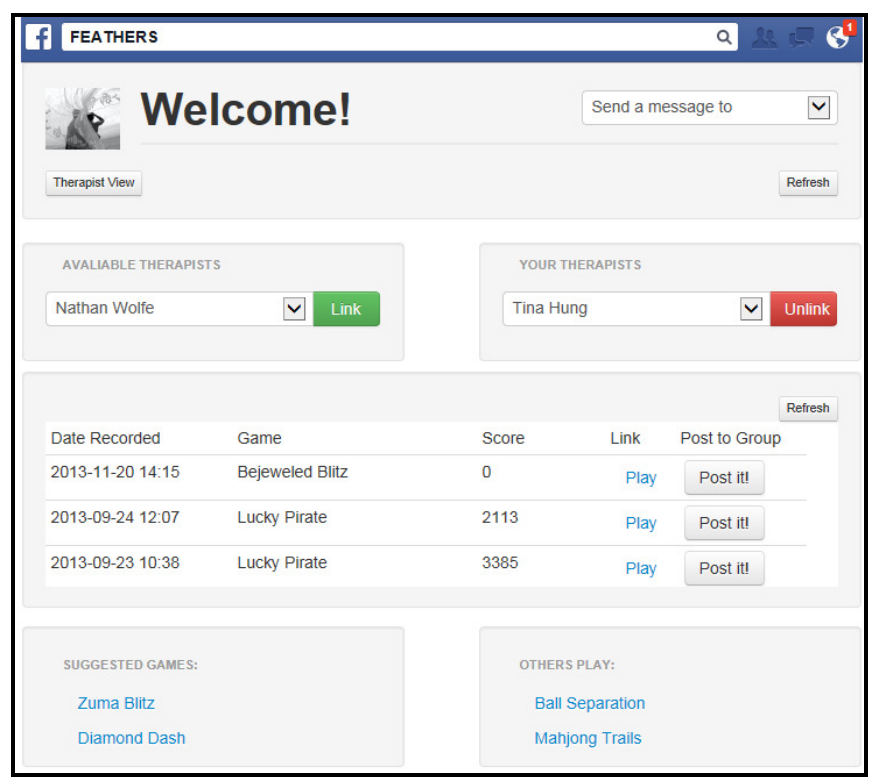

Fig. 4. Facebook Application for Target Populations

clinics. The UBC Research Ethics Board approved this study, and all participants provided written informed consent.

\section{Cognitive Walkthrough and Think Aloud Methods}

Subjects were divided into groups of 2-3 users to evaluate the applications using the Cognitive Walkthrough (CW) method. This usability tool provides a means to evaluate early working prototypes without comprehensive empirical user testing. The main goal in $\mathrm{CW}$ testing is to explore the degree to which the application supports exploratory learning [7]. Additionally, the Think Aloud (TA) method was employed to elicit verbal comments from users as they were trialing a system [8]. In our tests, the TA method was used to record the users' impressions on the different modes and tracking technologies while completing the different $\mathrm{CW}$ tasks.

Inside the testing rooms, a moderator and a note taker were present throughout the duration of the trials. The sessions were audio recorded. The subjects were given a laptop, the two motion tracking cameras, two customized controllers, and a printed walkthrough of 11 tasks (Table I), further divided into single actions that resulted in a total of 23 steps that the users had to complete. After each action was completed, the moderator asked the following questions:

- Will the users be trying to produce whatever effect the action has?

- Will users be able to notice that the correct action is available?

- Once users find the correct action at the interface, will they know that it is the right one for the effect they are trying to produce?

- After the action is taken, will users understand the feedback they get?

All users in the group answered these questions, and in cases of disagreement between group members, a note was made in the test records. All tasks were evaluated this way except for the third action in Task 7, in which all users were

(C) 2014 IEEE. Personal use of this material is permitted. Permission from IEEE must be obtained for all other uses, in any current or future media, including reprinting/republishing this material for advertising or promotional purposes, creating new collective works, for resale or redistribution to servers or lists, or reuse of any copyrighted component of this work in other works. 
TABLE I. COGNITIVE WALKTHORUGH RESULTS

\begin{tabular}{|c|c|c|c|}
\hline Task & Description & Action & $\begin{array}{l}\text { Failure } \\
\text { Stories }\end{array}$ \\
\hline 1 & $\begin{array}{l}\text { Log into the Facebook patient/therapist } \\
\text { application }\end{array}$ & 1 & $75 \%$ \\
\hline 2 & $\begin{array}{l}\text { Connect to a therapist using the } \\
\text { Facebook application }\end{array}$ & 1 & $75 \%$ \\
\hline \multirow{2}{*}{3} & \multirow{2}{*}{$\begin{array}{c}\text { Send message to therapist using the } \\
\text { Facebook application }\end{array}$} & 1 & $25 \%$ \\
\hline & & 2 & $75 \%$ \\
\hline 4 & $\begin{array}{l}\text { Start a game from the patient/therapist } \\
\text { application }\end{array}$ & 1 & $50 \%$ \\
\hline \multirow{2}{*}{$5 \mathrm{a}$} & \multirow{2}{*}{ Open FEATHERS Kinect Application } & 1 & $50 \%$ \\
\hline & & 2 & $0 \%$ \\
\hline \multirow{3}{*}{$5 b$} & \multirow{3}{*}{$\begin{array}{l}\text { Configure FEATHERS Kinect } \\
\text { Application }\end{array}$} & 1 & $0 \%$ \\
\hline & & 2 & $25 \%$ \\
\hline & & 3 & $25 \%$ \\
\hline \multirow{2}{*}{$6 a$} & \multirow{2}{*}{$\begin{array}{l}\text { Open FEATHERS PlayStation } \\
\text { Application }\end{array}$} & 1 & $25 \%$ \\
\hline & & 2 & $0 \%$ \\
\hline \multirow{3}{*}{$6 b$} & \multirow{3}{*}{$\begin{array}{c}\text { Configure FEATHERS PlayStation } \\
\text { Application }\end{array}$} & 1 & $25 \%$ \\
\hline & & 2 & $50 \%$ \\
\hline & & 3 & $25 \%$ \\
\hline $6 \mathrm{c}$ & Start tracking FEATHERS controllers & 1 & $25 \%$ \\
\hline \multirow{2}{*}{7} & \multirow{2}{*}{ Play game using hand motions } & 1 & $75 \%$ \\
\hline & & 2 & $0 \%$ \\
\hline \multirow{5}{*}{8} & \multirow{5}{*}{$\begin{array}{l}\text { Review game scores on Facebook } \\
\text { patient/therapist application }\end{array}$} & 1 & $0 \%$ \\
\hline & & 2 & $0 \%$ \\
\hline & & 3 & $0 \%$ \\
\hline & & 4 & $50 \%$ \\
\hline & & 5 & $50 \%$ \\
\hline
\end{tabular}

required to take turns playing the first level of a simple Facebook game ("Lucky Pirate", OUAT Entertainment). Subjects played the game using the two bimanual modes with each of the two tracking devices.

In addition to the $\mathrm{CW}$ questions, all users answered a post-test questionnaire that included multiple-option, Likert-type, and open-ended questions to evaluate their preferences and overall experience.

\section{Thematic Analysis}

Two researchers independently conducted a qualitative analysis of the verbatim transcriptions of Task 7. For this task we used a thematic analysis approach [9]. We investigated the following question: "What are the factors that facilitate and impede the use of technology in rehabilitation?"

\section{RESULTS AND DISCUSSION}

\section{A. Cognitive Walkthrough}

The CW results were analyzed to identify the success and failure stories of each action. If the answer to any of the four questions was "no", the action was classified as a failure story. If all the questions in one action received positive answers, this was considered a success story. The proportion of actions that were categorized as failure stories is shown in Table I.

The participants' comments on each of the failure stories were analyzed to identify which aspects of the applications needed improvement. In addition to the application-specific comments that we received from the $\mathrm{CW}$ testing (e.g., "make

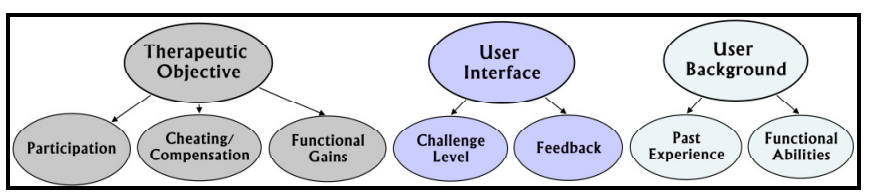

Fig. 5. Thematic Analysis Themes

the FEATHERS icon bigger"), we also obtained more general comments that can be used as a design guideline for computer interfaces developed for people with a combination of cognitive, visual, and physical impairments. For example, adding large visual feedback to every action taken, using minimal and descriptive labels, alphabetically ordering the lists for easier spotting, and minimizing the number of available actions.

\section{B. Thematic Analysis}

The themes and sub-themes that resulted from the Thematic Analysis are presented in Fig. 5, and are explained in the following section.

\section{1) Therapeutic Objective}

Participants emphasized the importance of ensuring the technology would be used to promote therapeutic movements. Comments like: "Right, right... So it's really smart! It's a functional fun way for them to use both hands together, without realizing that's... it's kind of like a therapy... mode..." reflected the similarity between the required control movements and current rehabilitation strategies. Moreover, the delivery of therapy in the form of games gives the opportunity of introducing these strategies to therapy clients in an engaging and motivating manner.

An increase in the level of interaction between clients and their peers (with and without disabilities) and a potential reintegration into society are important aspects of what therapists expect from the use of technology. While therapists were playing the video games, they appreciated the fact that they could compete against their colleagues to achieve higher scores: "Yeah, I am just trying to understand the game because I am very competitive. That's all, I like to win when I play this. "Using platforms like Facebook could facilitate the proliferation of social interactions, while providing a medium in which the users' physical limitations remain unnoticed and only their game scores are used to compete against other users, thus increasing the chances of users socializing not only with peers with similar disabilities, but also with non-disabled users.

All tested groups were interested in finding ways to cheat the systems by using compensatory movements. Compensatory movements can lead to pain, a reduction of a joint's range of motion and a pattern of "learned non-use" [10]. As a result, therapists seek to avoid the repetition of these movements, especially if their clients are using these systems in unsupervised settings, like their homes. Comments like: "But you see... I am still compensating with my body," led to a discussion about implementing strategies to detect compensatory movements and using the feedback capabilities of the systems to alert their clients about the use of these movements.

(C) 2014 IEEE. Personal use of this material is permitted. Permission from IEEE must be obtained for all other uses, in any current or future media, including reprinting/republishing this material for advertising or promotional purposes, creating new collective works, for resale or redistribution to servers or lists, or reuse of any copyrighted component of this work in other works. 


\section{2) User Interface}

The simplicity and ease of use of the interfaces were identified as major factors for the adoption of technology in therapy. Furthermore, the robustness of the tracking capabilities of the devices was one of the critical aspects that shaped the decision of therapists when choosing between the two motion tracking technologies, "I see people getting frustrated with this, if it is going to randomly open things."

The delivery of auditory, visual and haptic feedback to provide sensory input to the user was also mentioned by therapists. Suggestions included simple implementations such as receiving a haptic confirmation whenever the user pushed a button to click on an item on the screen: "I like the sensory feedback, because when I clicked it vibrated."

\section{3) User Background}

This theme was related to the past experiences and functional abilities of the therapists' clients. The trialed tracking technologies require users to continuously lift their arms against gravity, which results in physical fatigue. This issue was identified and commented on repeatedly throughout the sessions: "I find it quite tiring to keep my hands up." A possible solution is to introduce rest periods between game sessions that allow users to recover. In addition, games with short duration (e.g., mobile or online social games) could be employed, as they require the completion of simple objectives in a fixed amount of time.

System customization based on each user's initial physical capabilities is important. As the level of disability among clients varies, therapists are interested in being able to modify the parameters of the systems. This also applies for the case where clients start to improve over time. As a result, rehabilitation systems should have simple and clear interfaces that allow therapists and clients with different levels of computer skill to change the game and interface parameters.

\section{Post-test Questionnaire}

The eleven participants reported via post-test questionnaires on their experiences with the applications and motion tracking technologies. The results show that more than $72.7 \%$ of the participants agreed on the ease-of-use and simplicity of the Facebook application, and $90.9 \%$ enjoyed playing games using the motion tracking interfaces. Most (54.5\%) of the subjects preferred the PlayStation system over the Kinect system (18.2\%), while the rest liked both equally $(18.2 \%)$ or liked none $(9.1 \%)$. These results reflect the therapists' comments regarding the higher hand-tracking accuracy and simpler operation of the PlayStation system.

In terms of the bimanual control modes, $90.9 \%$ of the subjects agreed that using the VS mode was easy, while only $36.4 \%$ did for the PMS mode. A few therapists recommended the use of the latter mode for clients with higher upper limb functionality. The therapists were also asked which control mode they would prefer to use: $45.5 \%$ preferred the VS mode, $18.2 \%$ the PMS mode, and $36.3 \%$ both.

It was also found that $64.3 \%$ of therapists who participated in the experiment currently use computers, tablets or game consoles for gaming in rehabilitation sessions, while $35.7 \%$ do not use any technology for playing games. Thus, with $60.0 \%$ of the participants willing to recommend the developed applications for their clients' home-based rehabilitation programs, the use of this technology in rehabilitation seems promising.

\section{CONCLUSIONS}

The results from this phase of the project will guide the next design iteration of the developed applications. The failure stories from the Cognitive Walkthrough established the need for a step-by-step GUI in which visual feedback is given for every action taken by the user. In addition, the themes that emerged from the Thematic Analysis identified potential areas that have not been addressed by the current applications, i.e., reducing compensatory motions, customizing motion modes, and introducing rest periods. The next step for the project will be conducting user testing of the revised applications with the target populations. In addition, we will develop the required algorithms to analyze the kinematic data obtained by the systems to assess training effectiveness. The final phase of the project, will be a 6-month study in which users will follow a home rehabilitation program employing these novel systems.

\section{ACKNOWLEDGMENT}

The staff from the BC Centre for Ability and from Abilities Neurological Rehabilitation; Reality Controls; Mimi Law, Justin Hay, Alida Verster, Nathan Wolfe and Yujie Yang.

\section{REFERENCES}

[1] J.C. Andersen, A. Majnemer, K. O’Grady, and A. M. Gordon, "Intensive upper extremity training for children with hemiplegia: from science to practice.," Semin. Pediatr. Neurol., vol. 20, no. 2, pp. 100-5, Jun. 2013.

[2] W. Liu, S. McCombe Waller, T. M. Kepple, and J. Whitall, "Compensatory arm reaching strategies after stroke: induced position analysis.," J. Rehabil. Res. Dev., vol. 50, no. 1, pp. 71-84, Jan. 2013.

[3] S. K. Tatla, K. Sauve, N. Virji-Babul, L. Holsti, C. Butler, and H. F. M. Van der Loos, "Evidence for outcomes of motivational rehabilitation interventions for children and adolescents with cerebral palsy: an American Academy for Cerebral Palsy and Developmental Medicine systematic review.," Dev. Med. Child Neurol., vol. 55, no. 7, pp. 593-601, Jul. 2013.

[4] K. Lohse, N. Shirzad, A. Verster, N. Hodges, and H. F. M. Van der Loos, "Video games and rehabilitation: using design principles to enhance engagement in physical therapy.," J. Neurol. Phys. Ther., vol. 37, no. 4, pp. 166-75, Dec. 2013.

[5] RREACH (Robotics for Rehabilitation Exercise and Assessment in Collaborative Healthcare) Lab, "FEATHERS Project," 2014. [Online]. Available: http://caris.mech.ubc.ca/feathers/project-summary/.

[6] S. H. L. McAmis and K. B. Reed, "Simultaneous Perception of Forces and Motions Using Bimanual Interactions," IEEE Trans. Haptics, vol. 5, no. 3, pp. 220-230, 2012.

[7] J. Rieman, M. Franzke, and D. Redmiles, "Usability evaluation with the cognitive walkthrough," Conf. companion Hum. factors Comput. Syst. - CHI '95, pp. 387-388, 1995.

[8] C. Lewis and J. Rieman, Task-centered User Interface Design: A Practical Introduction. University of Colorado, Boulder, Department of Computer Science, 1993, p. 138.

[9] V. Braun and V. Clarke, "Using thematic analysis in psychology," Qual. Res. Psychol., no. November 2013, pp. 37-41, 2006.

[10] M. F. Levin, J. A. Kleim, and S. L. Wolf, "What do motor 'recovery' and 'compensation' mean in patients following stroke?," Neurorehabil. Neural Repair, vol. 23, no. 4, pp. 313-9, May 2009.

(C) 2014 IEEE. Personal use of this material is permitted. Permission from IEEE must be obtained for all other uses, in any current or future media, including reprinting/republishing this material for advertising or promotional purposes, creating new collective works, for resale or redistribution to servers or lists, or reuse of any copyrighted component of this work in other works. 